University of Texas at El Paso

ScholarWorks@UTEP

$11-2018$

\title{
Relativistic Effects Can Be Used to Achieve a Universal Square- Root (Or Even Faster) Computation Speedup
}

Olga Kosheleva

The University of Texas at El Paso, olgak@utep.edu

Vladik Kreinovich

The University of Texas at El Paso, vladik@utep.edu

Follow this and additional works at: https://scholarworks.utep.edu/cs_techrep

Part of the Computer Sciences Commons, and the Mathematics Commons Comments:

Technical Report: UTEP-CS-18-82

\section{Recommended Citation}

Kosheleva, Olga and Kreinovich, Vladik, "Relativistic Effects Can Be Used to Achieve a Universal SquareRoot (Or Even Faster) Computation Speedup" (2018). Departmental Technical Reports (CS). 1277. https://scholarworks.utep.edu/cs_techrep/1277

This Article is brought to you for free and open access by the Computer Science at ScholarWorks@UTEP. It has been accepted for inclusion in Departmental Technical Reports (CS) by an authorized administrator of ScholarWorks@UTEP.For more information, please contact Iweber@utep.edu. 


\title{
Relativistic Effects Can Be Used to Achieve a Universal Square-Root (Or Even Faster) Computation Speedup
}

\author{
Olga Kosheleva and Vladik Kreinovich \\ University of Texas at El Paso \\ $500 \mathrm{~W}$. University \\ El Paso, TX 79968 \\ olgak@utep.edu, vladik@utep.edu
}

\begin{abstract}
In this paper, we show that special relativity phenomenon can be used to reduce computation time of any algorithm from $T$ to $\sqrt{T}$. For this purpose, we keep computers where they are, but the whole civilization starts moving around the computer - at an increasing speed, reaching speeds close to the speed of light. A similar square-root speedup can be achieved if we place ourselves near a growing black hole. Combining the two schemes can lead to an even faster speedup: from time $T$ to the 4 -th order root $\sqrt[4]{T}$.
\end{abstract}

\section{Formulation of the Problem}

Need for fast computations. At first glance, the situation with computing speed is very good. The number of computational operations per second has grown exponentially fast, and continues to grow. Faster and faser high performance computers are being designed and built all the time, and the only reason why they are not built even faster is the cost limitations.

However, while, because of this progress, it has indeed become possible to solve many computational problems which were difficult to solve in the past, there are still some challenging practical problems that cannot yet been solved now. An example of such a problem is predicting where a tornado will go in the next 15 minutes. At present, this tornado prediction problem can be solved in a few hours on a high performance computer, but by then, it will be too late. As a result, during the tornado season, broad warning are often so frequent that people often ignore them - and fall victims when the tornado hits their homes.There are many other problems like this.

What can we do - in addition to what is being done. Computer engineers and computer scientists are well aware of the need for faster computations, 
so computer engineers are working on new hardware that will enable faster computations, and computer scientists are developing new faster algorithms for solving different problems. Some of the hardware efforts are based on the same physical and engineering principles on which the current computers operate, some efforts aim to involve different physical phenomena - such as quantum computing (see, e.g., [1]).

Can we use other physical phenomena as well? We are talking about speeding up computations, i.e., about time, so a natural place to look for such physical phenomena is to look for physical effects that change the rate of different physical processes, i.e., make them run faster or slower.

What we do in this paper. This is what we will do in this paper: we will show how physical phenomena can be used to further speed up computations.

\section{Physical Phenomena That Change the Rate of Physical Processes - and How to Use Them to Speed Up Computations}

Physical phenomena that change the rate of physical processes: a brief reminder. Unfortunately for computations, there are no physical processes that speed up all physical processes, but there are two physical processes that slow down all physical processes; see, e.g., [2, 5].

First, according to Special Relativity Theory, if we travel with some speed $v$, then all the processes slow down. The proper time interval $s$-i.e., the time interval registered by the observer moving with the speed $v$-is related to the time interval $t$ measured by the immobile observer by the formula

$$
s=t \cdot \sqrt{1-\frac{v^{2}}{c^{2}}}
$$

where $c$ denotes the speed of light. The closer the observer's speed $v$ to the speed to the speed of light $c$, the larger this slow-down.

Second, according to General Relativity Theory, in the gravitational field, time also slows down. For immobile observer, the proper time interval $s$ is equal to $s=\sqrt{g_{00}} \cdot t$, where $g_{00}$ is the 00-component of the metric tensor $g_{i j}$ that describes the geometry of space-time. In the spherically symmetric (Schwarzschild) solution, we have $g_{00}=1-\frac{r_{s}}{r}$, where $r$ is the distance from the center of the gravitating body and $r_{s} \stackrel{\text { def }}{=} \frac{2 G \cdot M}{c^{2}}$, where $G$ is the gravitational constant and $M$ is the mass of the central body.

Both slow-down effects have been experimentally confirmed with high accuracy.

How we can use these phenomena to speed up computations. If these phenomena would speed up all the processes, then it would be easy to speed up 
computations: we would simply place the computers in a body moving with a high speed and/or located in a strong gravitational field, and we would this get computations faster.

In reality, these phenomena slow down all the processes, not speed them up. So, if we place computers in such a slowed-time environment, we will only slow down the computations. However, we can speed up computations if we do the opposite: namely, keep computers in a relatively immobile place with a reasonably low gravitational field, and place our whole civilization in a fast moving body and/or in a strong gravitational field. In this case, in terms of the computers themselves, computations will continue at the same speed as before, but since our time will be slowed down, we will observe much more computational steps in the same interval of proper time (i.e., time as measured by our slowed-down civilization).

In this paper, we analyze what speed up we can obtain in this way - by analyzing the above slowing-down physical phenomena one by one.

\section{Possible Special-Relativity Speed-Up: Analy- sis of the Problem and Resulting Formulas}

How to use special relativistic effects for a speed-up: reminder. To get a speed-up, we can place the computer at the center, and start moving around this computer at a speed close to the speed of light. Since we cannot immediately reach the speed of light or the desired trajectory radius, we need to gradually increase our speed and the radius. Let $v(t)$ denote our speed at time $t$, and let $R(t)$ denote the radius of our trajectory at moment $t$.

Analysis of the problem. According to the above formula (1), a change $d s$ in proper time is related to the change $d t$ in coordinate time (as measured by the computer clock) is $d s=d t \cdot \sqrt{1-\frac{v^{2}(t)}{c^{2}}}$. To make civilization with rest energy $E_{0}$ move with this speed, we need the energy

$$
E(t)=\frac{E_{0}}{\sqrt{1-\frac{v^{2}(t)}{c^{2}}}} .
$$

Thus, we can say that $d s=d t \cdot \frac{E_{0}}{E(t)}$.

The possibility to travel is limited by the need to keep acceleration experienced by all moving persons at the usual Earth level $g_{0}$. When a body follows a circular orbit with velocity $v(t)$ and radius $R(t)$, it experiences acceleration $\frac{d^{2} x}{d t^{2}}=\frac{v^{2}(t)}{R(t)}$. Since the velocity $v(t)$ is close to the speed of light $v(t) \approx c$, we conclude that $\frac{d^{2} x}{d t^{2}}=\frac{c^{2}}{R(t)}$. Substituting $d t=d s \cdot \frac{E(t)}{E_{0}}$ into this formula, we 
conclude that

$$
\frac{E_{0}^{2}}{E^{2}(t)} \cdot \frac{d^{2} x}{d s^{2}}=\frac{c^{2}}{R(t)}
$$

Here, the experienced acceleration $\frac{d^{2} x}{d s^{2}}$ should be equal to the usual Earth acceleration $g_{0}$, thus

$$
\frac{E_{0}^{2}}{E^{2}(t)} \cdot g_{0}=\frac{c^{2}}{R(t)}
$$

Thus, the speed-up $\frac{E(t)}{E_{0}}$ can be determined as

$$
\frac{E(t)}{E_{0}}=c \cdot \sqrt{\frac{R(t)}{g_{0}}} .
$$

The larger $R(t)$, the larger the speed-up. All the speeds are limited by the speed of light, thus, we have $R(t) \leq v_{0} \cdot t$, where $v_{0}<c$ is the speed with which we increase the radius. To increase the speed-up effect, let us consider the case when $R(t)=v_{0} \cdot t$. In this case, the speedup has the form

$$
\frac{E(t)}{E_{0}}=C \cdot \sqrt{t}
$$

where we denoted $C \stackrel{\text { def }}{=} \frac{c \cdot \sqrt{v_{0}}}{\sqrt{g_{0}}}$. Thus, we get

$$
\frac{d s}{d t}=\frac{E_{0}}{E(t)}=C^{-1} \cdot t^{-1 / 2},
$$

hence $d s=C^{-1} \cdot d t \cdot t^{-1 / 2}$. Integrating both sides, we conclude that $s=2 C^{-1} \cdot \sqrt{t}$. Thus, we arrive at the following speed-up scheme.

Resulting speedup scheme. To speed up computations, we place computers where they are now, and start moving the whole civilization. At any given moment of time $t$, we move the civilization at a circle of radius $R(t)=v_{0} \cdot t$, for some pre-determined radial speed $v_{0}<c$.

The speed $v(t)$ is determined by the formula

$$
\frac{E_{0}^{2}}{E^{2}(t)}=1-\frac{v^{2}(t)}{c^{2}}=\frac{c^{2}}{R(t) \cdot g_{0}}=\frac{c^{2}}{v_{0} \cdot g_{0} \cdot t}
$$

hence

$$
v(t)=c \cdot \sqrt{1-\frac{c^{2}}{v_{0} \cdot g_{0} \cdot t}} .
$$

In this scheme, the coordinate time $t$ is related to the proper time $s$ by the formula $s=2 C^{-1} \cdot \sqrt{t}$, where $C=\frac{c \cdot \sqrt{v_{0}}}{\sqrt{g_{0}}}$. Thus, we indeed get a square-root speedup. 
This is all we can get. Please note that this square root speedup is all we can gain: a further speedup would require having accelerations much higher than our usual level $g_{0}$.

How realistic is this scheme? How big a radius do we need to reach a reasonable speedup? As we will show, the corresponding radius is - by astronomical standards - quite reasonable. Indeed, for $E(t) \approx E_{0}$, the above formulas relating $E(t)$ and $R(t)$ leads to

$$
R(t)=\frac{c^{2}}{g_{0}} \approx \frac{\left(3 \cdot 10^{8} \mathrm{~m} / \mathrm{sec}\right)^{2}}{10 \mathrm{~m} / \mathrm{sec}^{2}}=9 \cdot 10^{15} \mathrm{~m} .
$$

This radius can be compared with a 1 light year - the distance that the light travels in 1 year - which is equal to

$$
\approx\left(3 \cdot 10^{8} \mathrm{~m} / \mathrm{sec}\right) \cdot\left(3 \cdot 10^{7} \mathrm{sec} / \text { year }\right) \cdot(1 \text { year })=9 \cdot 10^{15} \mathrm{~m},
$$

so for $E(t)=E_{0}$, the radius should be about 1 light year. With a speed-up $E(t) / E_{0}$, the radius grows as the square of this speed-up. So:

- to get an order of magnitude (10 times) speedup, we need an orbit of radius $10^{2}=100$ light years - reaching to the nearest stars;

- to get a two orders of magnitude (100 times) speedup, we need an orbit of radius $100^{2}=10^{4}$ light years - almost bringing us to the edge of our Milky Way Galaxy;

- to get a three orders of magnitude (1000 times) speedup, we need an orbit of radius $1000^{2}=10^{6}$ light years;

- with an orbit of the same radius as the radius of the University $R(t) \approx 20$ billion $=2 \cdot 10^{10}$ light years, we can get $\sqrt{2 \cdot 10^{10}} \approx 1.5 \cdot 10^{5}$ speedup more than hundred thousand times speedup.

This is similar to a quantum speedup. The above square root speedup is similar to the speedup of Grover's quantum algorithm for search in an unsorted array $[3,4,1]$; the difference is that:

- in quantum computing, the speedup is limited to search in an unsorted array, while

- in the above special-relativity scheme, we get the same speedup for all possible computations.

Comment. In Russia - where we are from - to ring the church bells, the monks move the bell's "tongue". In Western Europe, they move the bell itself. This example is often used in Russian papers on algorithm efficiency, with an emphasis on the fact that, in principle, it is possible to use a third way to ring the bell: by shaking the whole bell tower. In these papers, this third way is mentioned 
simply as a joke, but, as the above computations show, this is exactly what we are proposing here: since we cannot reach a speedup by making the computer move, we instead leave the computers intact and move the whole civilization.

Speculation. How can we check whether an advanced civilization is already using this scheme? In this scheme, a civilization rotates around a center, increasing its radius as it goes - i.e., follows a spiral trajectory. In this process, to remaining accelerating, the civilization needs to gain more and more kinetic energy $E(t)$. The only way to get this energy is to burn all the burnable matter that it encounters along its trajectory. As a result, along the trajectory, where the matter has been burned, we have low-density areas.

Thus, as a trace of such a civilization, we are left with a shape in which there are spiral-shaped low-density areas starting form some central point. But this is exactly how our Galaxy - and many other spiral galaxies - look like. So maybe this is how spiral galaxies acquired their current shape?

\section{Possible General-Relativity Speed-Up: Anal- ysis of the Problem and Resulting Formulas}

Idea. If we keep the computers were they are now, and place the whole civilization in a strong gravitational field, then our proper time will slow down. Thus, the computations that take the same coordinate time $t$ will require, in terms of our proper time $s$, much fewer seconds.

Analysis of the problem. According to the Schwarzschild's formula for the gravitational field of a symmetric body of mass $M(t)$ at a distance $R(t)$ from the center, for an immobile body, we have $d s^{2}=g_{00} \cdot d t^{2}$, where $g_{00}(t)=$ $1-\frac{2 G \cdot M(t)}{c^{2} \cdot R(t)}$. So, the slow-down $\varepsilon(t) \stackrel{\text { def }}{=} \frac{d s}{d t}$ is equal to

$$
\varepsilon(t)=\sqrt{g_{00}(t)}=\sqrt{1-\frac{2 G \cdot M(t)}{c^{2} \cdot R(t)}} .
$$

We want a good speedup, with $\varepsilon(t) \approx 0$, so we should have $M(t) \approx \frac{c^{2} \cdot R(t)}{2 G}$. The coordinate acceleration is equal to $\frac{G \cdot M(t)}{R^{2}(t)}$ :

$$
\frac{d^{2} x}{d t^{2}}=\frac{G \cdot M(t)}{R^{2}(t)}
$$

Substituting the above expression for $M(t)$ into this formula, we conclude that

$$
\frac{d^{2} x}{d t^{2}}=\frac{c^{2} \cdot R(t)}{2 R^{2}(t)}=\frac{c^{2}}{2 R(t)} .
$$


The observed acceleration thus takes the form

$$
\frac{d^{2} x}{d s^{2}}=\frac{d^{2} x}{d t^{2}} \cdot\left(\frac{d t}{d s}\right)^{2}=\frac{c^{2}}{2 R(t)} \cdot \frac{1}{\varepsilon^{2}(t)} .
$$

This acceleration should be equal to the usual Earth's acceleration $g_{0}$ :

$$
\frac{c^{2}}{2 R(t)} \cdot \frac{1}{\varepsilon^{2}(t)}=g_{0}
$$

thus $\varepsilon(t)=\frac{c}{\sqrt{2 R(t) \cdot g_{0}}}$. So, to get faster and faster computations, we need to constantly increase $R(t)$ - and thus, increase the mass $M(t)$ which is proportional to $R(t)$.

Similarly to the special relativity case, $R(t)$ cannot grow faster than linearly, so we have $R(t)=v_{0} \cdot t$. In this case, the speed-up is proportional to $\varepsilon(t) \sim t^{-1 / 2}$, and, similarly to the special relativity case, we get a square-root speedup.

Resulting speedup scheme. To speed up computations, we place computers where they are now, and move at a distance $R(t)=v_{0} \cdot t$ from a body of a constantly increasing mass $M(t)=\frac{c^{2} \cdot R(t)}{2 G}$, where $G$ is the gravitational constant - and we ourselves need to continually increase the corresponding mass.

In this scheme, we also get a square-root speedup.

This is all we can get. Please note that, similarly to the special relativity scheme, this square root speedup is all we can gain: a further speedup would require having accelerations much higher than our usual level $g_{0}$.

Astrophysical comment. It is known that there is a threshold of masses after which a body with a sufficiently large mass becomes a black hole. Thus, in this scheme, after some time, the civilization is close to a black hole.

\section{Ideally, We Should Use Both Speedups}

Moving at a speed close to the speed of light decreases the proper time from the original value $t$ to a much smaller amount $s \sim \sqrt{t}$. Similarly, a location near a black hole also decreases the observable computation time to a square root of its original value.

Thus, if we combine these two schemes - i.e., place ourselves near an everincreasing black hole and move (together with this black hole) at a speed close to the speed of light, we will get both speedups, i.e., we will replace the perceived computation time from $T$ to $\sqrt{\sqrt{T}}=\sqrt[4]{T}$.

\section{Acknowledgments}

This work was partially supported by the US National Science Foundation via grant HRD-1242122 (Cyber-ShARE Center of Excellence). 


\section{References}

[1] M. Nielsen and I. Chuang, Quantum Computation and Quantum Information, Cambridge University Press, Cambridge, 2000.

[2] R. Feynman, R. Leighton, and M. Sands, The Feynman Lectures on Physics, Addison Wesley, Boston, Massachusetts, 2005.

[3] L. K. Grover, "A fast quantum mechanical algorithm for database search", Proceedings of the 28th ACM Symposium on Theory of Computing, 1996, pp. 212-219.

[4] L. K. Grover, "Quantum mechanics helps in searching for a needle in a haystack", Physical Reviews Letters, 1997, Vol. 79, No. 2, pp. 325-328.

[5] K. S. Thorne and R. D. Blandford, Modern Classical Physics: Optics, Fluids, Plasmas, Elasticity, Relativity, and Statistical Physics, Princeton University Press, Princeton, New Jersey, 2017. 